\title{
Interpretation of Chinese and American Guidelines for Diagnosis and Treatment of Thyroid Diseases During Pregnancy
}

\author{
Jiang Bohua ${ }^{1}$, Tao Xinli ${ }^{2,}$, , Ouyang Zhenbo ${ }^{3}$, Yan Jiane ${ }^{1}$ \\ ${ }^{1}$ Changsha County Maternal and Child Health Care Hospital, Changsha, China \\ ${ }^{2}$ Department of Gynecology, Xiangzhou District People's Hospital of Xiangyang City, Xiangyang, China \\ ${ }^{3}$ Department of Gynecology, Second People's Hospital of Guangdong Province, Guangzhou, China
}

\section{Email address:}

taoxinli77@163.com (Tao Xinli)

${ }^{*}$ Corresponding author

\section{To cite this article:}

Jiang Bohua, Tao Xinli, Ouyang Zhenbo, Yan Jiane. Interpretation of Chinese and American Guidelines for Diagnosis and Treatment of Thyroid Diseases During Pregnancy. Science Discovery. Vol. 9, No. 4, 2021, pp. 133-138. doi: 10.11648/j.sd.20210904.11

Received: April 6, 2021; Accepted: May 20, 2021; Published: May 24, 2021

\begin{abstract}
More and more studies have found that thyrotoxicosis and hypothyroidism are related to adverse pregnancy outcomes, and maternal thyroid diseases also have a certain impact on fetal development. Drugs used to treat maternal hyperthyroidism can pass through the placenta and affect fetal thyroid development and thyroid function, and may cause fetal development malformations . Due to the lack of in-depth knowledge of this disease, timely screening, medical consultation and treatment rates for thyroid diseases during pregnancy are relatively low. Like this, if the treatment is not timely, it may cause irreversible effects on the pregnancy process and the fetus, such as increasing the risk of high blood pressure during pregnancy, miscarriage, premature delivery, low birth weight, stillbirth, thyroid crisis and maternal congestive heart failure, and impairing the neurological and intellectual development of offspring. This article intends to provide a reference for clinicians in the standardized diagnosis and treatment of thyroid diseases during pregnancy and postpartum through a comparative analysis of the latest guidelines for the diagnosis and treatment of thyroid diseases during pregnancy in China and the United States in 2020. It is hoped that through this article, various related complications and risks of mothers, fetuses and pregnancy caused by abnormal thyroid function will be reduced, and the quality of the population will be improved.
\end{abstract}

Keywords: Pregnancy, Thyroid Disease, Diagnosis and Treatment Norms, Guidelines

\section{中美妊娠期甲状腺疾病诊疗指南的解读}

\author{
姜柏华 ${ }^{1}$, 陶金丽 ${ }^{2 *}$, 欧阳振波 $^{3}$, 颜件娥 $^{1}$ \\ 1长沙县妇幼保健院, 长沙, 中国 \\ 2襄阳市襄州区人民医院妇科, 襄阳, 中国 \\ 3 广东省第二人民医院妇科, 广州, 中国
}

\section{邮箱}

taoxinli77@163.com（陶金丽）

摘要: 越来越多的研究发现, 甲状腺毒症和甲状腺功能楲退与不良妊娠结局相关, 而且母体甲状腺疾病对胎儿发育也 存在一定的影响。治疗母体甲状腺功能六进的药物可以穿过胎盘并影响胎儿甲状腺发育及甲状腺功能, 并且可能导致 胎儿发育畸形。由于对此疾病的认识程度欠深入, 妊娠期甲状腺疾病的及时篮查、就诊率及治疗率均较低, 这可能对 妊娠过程及胎儿造成不可逆的影响, 如增加妊娠期高血压、流产、早产、低出生体重儿、死胎、甲状腺危象和孕产妇 
充血性心力衰竭等风险, 及损害后代神经智力发育。本文主要通过对2020年中、美两国最新的妊娠期甲状腺疾病诊疗 指南进行对比分析及总结, 为临床医师们对妊娠期及产后甲状腺疾病的规范化诊治提供参考, 从而减少因甲状腺功能 异常而导致的母体、胎儿及妊娠过程中的各种相关并发症及风险，提高人口素质。

关键词：妊娠，甲状腺疾病，诊治规范，指南

\section{1. 引言}

越来越多的研究发现, 甲状腺毒症和甲状腺功能减 退与不良妊娠结局有关，而且母体甲状腺疾病对胎儿发 育也存在一定的影响, 抗甲状腺药物可通过胎盘从而影 响胎儿甲状腺发育等[1]。由于对此疾病的认识程度欠深 入, 妊娠期甲状腺疾病的及时篩查、就诊率及治疗率均 较低, 将对妊娠过程及胎儿造成不可逆的影响。因此, 为规范其诊治, 2019年中华医学会围产医学分会和中华 医学会内分泌学分会联合发布了更新指南《妊娠和产后 甲状腺疾病诊治指南》(第2版) [2] (以下简称中国指南), 以替代2012年第1版指南。2020年美国妇产科医师学会 (ACOG) 也发布了此疾病的最新指南, 即《妊娠期甲 状腺疾病》[3]（以下简称美国指南）, 以替代2015年版 指南。因此, 为了更好地帮助临床医生们了解及规范妊 娠期及产后甲状腺疾病的诊治, 从而减少因甲状腺功能 异常而导致的母体、胎儿及妊娠过程中的各种相关并发 症及风险, 本文将对中美两国最新诊治指南进行对比分 析。

两指南均主要从妊娠期母体及胎儿甲状腺的生理变 化及相关问题, 和妊娠期及产后甲状腺疾病的诊疗两大部 分进行了详细的阐述。虽然在侧重点上存在细微差异, 但 总体相似, 本文主要从以上两大部分的十个小点分别进行 解读分析。

\section{2. 妊娠期母体甲状腺功能的变化及胎儿甲状腺 生理}

妊娠期母体甲状腺有较大的生理性改变, 易与母体 甲状腺异常相混淆。因此了解妊娠期母体甲状腺功能的 变化对妊娠期甲状腺疾病的诊治很有帮助。由于妊娠期 间细胞外液和血容量的增加, 母体甲状腺体积在妊娠晚 期增长约 $30 \%[4]$ 。此外, 在整个妊娠期间甲状腺激素水 平和甲状腺功能均存在一定变化 $[2,3]$ : (1)在雌激素 $\left(\mathrm{E}_{2}\right)$ 的刺激下, 甲状腺素结合球蛋白 ( TBG) 产生增加, 清 除减少。TBG自妊娠6 8周开始逐渐增加, 妊娠20周达高 峰, 较基础值增加1.5 2.0倍, 持续至分婏。 $\mathrm{TBG}$ 的增加 会导致总甲状腺素（TT4）浓度增加。(2)妊娠早期胎盘分 泌的 $\mathrm{hCG}$ 增加, 在妊娠 $8 \sim 10$ 周达到高峰, 因 $\mathrm{hCG}$ 的 $\alpha$ 亚单 位与促甲状腺激素 (TSH) 相似, 对 TSH受体有微弱的 刺激作用, 从而增加血清游离甲状腺素 (FT4) 水平, 抑 制下丘脑 TSH释放, 而导致早孕期TSH的减少。血清TSH 降低发生在妊娠8 14周, 10 12周达最低水平。(3)在妊娠 三个月后, TSH逐渐恢复至基线水平, 并在妊娠末期因 胎盘脱碘酶的产生而逐渐增加。(4)由于妊娠期免疫耐受,
甲状腺自身抗体滴度在妊娠后期逐渐下降，于20 30周降 低约 $50 \%$, 分娩后抗体滴度回升, 于产后 6 个月恢复至妊 娠前水平。

妊娠约 12 周时胎儿甲状腺开始摄碘及合成甲状腺激 素。母体T4在整个妊娠期均有转移至胎儿体内, 并对胎儿 的神经系统发育起着重要作用, 特别是在胎儿甲状腺出现 功能之前。母体抗甲状腺药物的使用及母体甲状腺抗体均 可对胎儿甲状腺功能造成一定的影响[3]。

\section{3. 妊娠期甲状腺功能相关指标的参考范围}

由于妊娠期甲状腺相关激素存在上述特异性变化, 以 及妊娠期TBG浓度的增加和白蛋白浓度的减少均会影响 免疫检测法结果, 因此妊娠期甲状腺激素不能用非妊娠期 的参考范围进行衡量。既往 $2.5 \mathrm{mIU} / \mathrm{L}$ 一度被视为妊娠早期 女性 TSH参考范围上限, 但越来越多的研究表明这可导致 过度诊断, 因而 $2.5 \mathrm{mIU} / \mathrm{L}$ 已不再是诊断妊娠期甲减的截断 值 [5], 最好的办法是各地区和单位建立试剂及妊娠期特异 性甲状腺功能指标参考范围。

中国指南推荐了三种建立妊娠期甲状腺相关激素 参考范围的方法: (1)在本单位或者本地区采取 NACB 推荐的方法, 建立方法和妊娠期 (早、中、晚期) 特 异的血清甲状腺功能指标参考范围; (2)采用指南提供 的试剂公司的甲状腺功能指标参考范围; (3) TSH可采 用普通人群参考范围上限降低 $22 \%$, 或采用 $4.0 \mathrm{mIU} / \mathrm{L}$ 作为参考范围上限; FT4下限根据普通人群的下限在孕 早期升高 7\%, 孕中期下降 $13 \%$, 孕晚期下降 $21 \%[2]$ 。 美国指南 [3]也推荐通过上述第一种方式获取妊娠期特 异性参考范围, 但当当地参考范围无法实现时, 在妊 娠早期, 可将 TSH的参考下限减少 $0.4 \mathrm{mU} / \mathrm{L}$, 参考上限 减少 $0.5 \mathrm{mU} / \mathrm{L}$ 。在妊娠中晚期, $\mathrm{TSH}$ 可使用非妊娠参考 范围。TT4和 TT3 的参考范围也应进行调整。妊娠 16 周 前, TT4和 TT3逐渐增加, 妊娠 16周后 TT4 和 TT3 的参 考上限可增加约 $50 \%$ 。

\section{4. 妊娠期甲状腺相关疾病}

妊娠期甲状腺功能异常相关疾病主要包括: 临床及亚 临床甲状腺功能减退症、妊娠期单纯低甲状腺素血症、妊 娠期甲状腺自身抗体阳性、妊娠期甲状腺毒症、妊娠期甲 状腺结节和甲状腺癌等, 这些疾病对妊娠过程及胎儿存在 不同程度的影响, 部分需要给与相应的治疗, 下文将进行 详细阐释。 


\section{1. 临床甲状腺功能减退症}

不少研究已明确妊娠期临床甲减会对妊娠造成不良 影响, 如增加妊娠期高血压、流产、早产、低出生体重儿 和死胎等风险及损害后代神经智力发育, 因此中美指南均 建议必须给予治疗。

中国指南推荐, 已患临床甲减的妇女需先将血清 TSH 控制在正常参考范围下限 $2.5 \mathrm{mU} / \mathrm{L}$ 水平后再计划妊娠; 临 床甲减妇女妊娠后LT4替代剂量需增加 $20 \%$ 30\%, 并根据 血清TSH及时调整LT4剂量; 而妊娠期临床甲减一旦确诊, 应立即治疗, 药物选择左甲状腺素（LT4）治疗。美国指 南建议对于孕前行LT4治疗的患者, 在确认妊娠后LT4剂 量增加 $25 \%$ 。对于LT4的治疗剂量, 中美指南给出不一样 的计算方法, 但在治疗剂量上相似。中国指南推荐的LT4

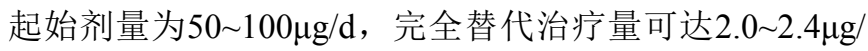
（kg.d）。美国指南推荐的起始剂量为 $1 \sim 2 \mu \mathrm{g} /(\mathrm{kg} . \mathrm{d})$ 或 $100 \mu \mathrm{g} / \mathrm{d}$, 尽快使得 TSH达标。对于妊娠期临床甲减的治疗 目标, 中美指南也给出了不同的计算方式。中国指南推荐 将 TSH控制在妊娠期特异性参考范围的下 $1 / 2$, 或小于 $2.5 \mathrm{mU} / \mathrm{L}$ 。美国指南建议将 $\mathrm{TSH}$ 控制在参考范围下限至 $2.5 \mathrm{mU} / \mathrm{L}$ 之间。此外, 对于妊娠期临床甲减的监测, 在妊 娠前半期中国指南（2 4周/次）推荐的监测频率高于美国

(4 6周/次), 此后相同（4 6周/次）。综上, 对于LT4 治疗剂量、TSH控制标准及监测频率上中美指南存在细微 的差异, 但总体在相似范围, 临床医生可根据自己的习惯 进行选择。

\section{2. 妊娠期亚临床甲状腺功能减退症（SCH）}

妊娠期 SCH对妊娠结局是否有影响存在一定的争议。 有研究表明妊娠期 SCH治疗与不良妊娠结局无明显相关 [6], 且其诊断和治疗并没有改善妊娠结局和后代的神经认 知功能 [7], 因此美国指南不推荐常规篎查及治疗。但另有 部分研究表明妊娠期SCH增加不良妊娠结局发生风险, 可 能与TPOAb 状态等因素有关 [8], 因此中国指南对此治疗 相对积极。中国指南推荐妊娠期SCH根据血清TSH水平和 $\mathrm{TPOAb}$ 是否阳性给予不同的治疗方案：(1) TSH $>$ 妊娠期参 考范围上限(或 $4.0 \mathrm{mU} / \mathrm{L}$ ), 无论 $\mathrm{TPOAb}$ 是否阳性, 均推荐 LT4 治疗; (2) TSH在 $2.5 \mathrm{mU} / \mathrm{L} \sim$ 妊娠期参考范围上限(或 $4.0 \mathrm{mU} / \mathrm{L})$, 伴TPOAb阳性, 考虑LT4治疗; TPOAb阴性, 不考虑 LT4 治疗; (3) TSH在妊娠期参考范围下限 (或 $0.1 \mathrm{mU} / \mathrm{L}) \sim 2.5 \mathrm{mU} / \mathrm{L}$, 不推荐LT4治疗, TPOAb阳性需监测 $\mathrm{TSH}, \mathrm{TPOAb}$ 阴性, 无需监测。妊娠期SCH的治疗药物、 治疗目标和监测频率与妊娠期临床甲减相同, LT4的治疗 剂量根据 TSH检测情况给予适当的起始剂量, 此剂量可能 小于妊娠期临床甲减。因此对于妊娠期 $\mathrm{SCH}$, 临床医生应 告知患者治疗的争议, 可适当放宽治疗指针, 结合孕妇的 具体情况及治疗意愿, 选择合适的治疗方案。

\section{3. 妊娠期单纯低甲状腺素血症}

单纯低甲状腺素血症又称低T4血症, 是指妊娠妇女血 清TSH水平正常、甲状腺自身抗体阴性, 且FT4水平低于 参考范围下限。低T4血症原因不明确, 碘缺乏、碘过量及 妊娠早期铁缺乏可能是导致低甲状腺素血症的原因 $[9,10]$ 。
而LT4治疗能否改善后代神经智力发育和不良妊娠结局, 其证据不足, 因此中国指南既不推荐也不反对在妊娠早期 给予LT4治疗, 但建议查找原因, 对因治疗。而美国指南 未对此进行推荐及建议。

\section{4. 妊娠期甲状腺自身抗体阳性}

甲状腺自身抗体阳性是指 $\mathrm{TPOAb}$ 或 $\mathrm{TgAb}$ 的滴度超 过参考范围上限, 不伴血清 TSH异常。对此类患者进行 LT4治疗能否改善妊娠结局并不十分明确, 因此中美两 国指南推荐存在差异。有meta分析表明, 甲状腺自身抗 体阳性组的流产风险升高[11]。因此中国指南认为对于 有不明原因流产史的妊娠期甲状腺自身抗体阳性者, LT4治疗可能有益, 且风险小, 可给予LT4 25 50 $\mu \mathrm{g} / \mathrm{d}$ 起始剂量治疗, 不推荐补硒治疗。此外也有研究表明, 对单纯 TPOAb 阳性妇女进行 LT4治疗并不能改善妊娠 结局, 因而美国指南不推荐对妊娠妇女进行 TPOAb或 $T g A b$ 常规检测, 而且不建议对单纯TPOAb阳性妇女进 行LT4治疗。

\section{5. 妊娠期甲状腺毒症}

妊娠期甲状腺毒症患病率约 $1 \%$, 其中临床甲六约 $0.4 \%$, 亚临床甲六约 $0.6 \%$ 。其病因主要有毒性弥漫性甲 状腺肿 (Graves病) 和妊娠一过性甲状腺毒症（GTT）， 分别占 $85 \%$ 和 $10 \%$, 此外还有结节性甲状腺肿、甲状腺高 功能腺瘤、甲状腺破坏以及外源性甲状腺激素过量补充 等。妊娠期甲状腺功能状态与妊娠结局密切相关, 甲六 控制不良与妊娠期高血压、流产、早产、低出生体重儿、 胎儿宫内生长受限、死胎、死产、甲状腺危象及孕产妇 充血性心力衰竭等相关。有研究表明胎儿暴露于过多的 母体甲状腺激素, 可能导致远期患癫㾁和神经行为异常 疾病的风险增加, 影响后代智力和脑皮质容量 $[12,13]$ 。 而且母体高水平甲状腺激素, 可通过胎盘进入胎儿体内, 导致胎儿甲六, 进而抑制胎儿垂体 TSH, 导致新生儿出 生后一过性中枢性甲减。此外, 若Graves病孕妇妊娠中 晚期促甲状腺素受体抗体 (TRAb) 水平明显升高, 胎儿 和新生儿甲六风险增加[14]。因此需要给予相应的监测和 治疗。

\subsection{1. 抗甲状腺药物选择}

妊娠期间应用抗甲状腺药物（ATD），母体发生肝毒 性、白细胞减少等副作用的几率相对较低, 但孕早期应用 ATD可增加胎儿致畸风险 $[15,16]$ 。抗甲状腺药物主要有2 种：甲颈咪唑（MMI）和丙硫氧嘧啶（PTU）。MMI相关 胎儿发育畸形主要是 “甲統咪唑相关的胚胎病”和皮肤发 育不全, 如鼻后孔闭锁、食道闭锁、颜面畸形等 $[16,17]$ 。 在对已知Graves病的5967个活产儿的回顾性分析中, 暴露 于MMI与暴露于PTU的患者相比, 胎儿畸形的风险增加了 两倍 $[18]$ 。妊娠6 10周是抗甲状腺药物导致出生缺陷的危 险窗口期, 如想完全避免ATD导致的先天畸形, 则应在致 畸关键期（妊娠6 10周）之前停药, 如必须使用, 在妊娠 前和妊娠早期优先选择PTU。妊娠中晚期, PTU和MMI均 可用于治疗甲状腺机能六进症。 


\subsection{2. 妊娠一过性甲状腺毒症（GTT）}

GTT指血清TSH低于妊娠期特异性参考范围下限(或 $0.1 \mathrm{mU} / \mathrm{L})$, FT4 > 妊娠期特异性参考范围上限, 排除其他 病因引起的甲六。GTT与胎盘分泌高水平的 $\mathrm{hCG}$, 刺激甲 状腺激素产生有关，多在妊娠14 18周恢复正常。中国指 南指出, GTT治疗以对症支持疗法为主, 纠正脱水及电解 质紊乱, 不建议给予ATD治疗, 如病情需要, 可以考虑应 用 $\beta$ 受体阻滞剂。妊娠剧吐患者常合并短暂甲六，但多无 症状, 抗甲状腺药物治疗无明显收益, 因此美国指南不建 议对妊娠剧吐患者进行甲功检测, 除非有明显甲立症状。 除此之外, 对于GTT美国指南未给与其他相关建议。

\subsection{3. 妊娠合并Graves病甲六}

Graves病是一种自身免疫性疾病, 常伴有 $\mathrm{TRAb}$ 升高, 导致甲状腺功能六进症, 其临床表现并不限于甲状腺, 而 是一种多系统的综合征。对于妊娠期Graves病患者, 需视 情况进行药物治疗, 此外除关注甲状腺功能外, 还需关注 $\mathrm{TRAb}$ 过高给胎儿及新生儿带来的潜在风险, 做好新生儿 监测。因此对此中国指南给出了详细推荐, 而美国指南仅 简单介绍了Graves病对母儿的危害, 对其治疗未进行详细 阐述, 临床医生可参照中国指南的推荐进行临床诊疗。

对于妊娠合并Graves病甲䒕的妇女, 中国指南给出的 详细推荐如下。（1）罹患Graves病甲六的妇女计划妊娠 时, 最好在甲状腺功能控制至正常并平稳后妊娠 (即治疗 方案不变的情况下，2次间隔至少一个月甲功测定在正常 范围），以减少妊娠不良结局。（2）如正在服用抗甲状 腺药物的备孕妇女已确诊妊娠, 可暂停药物并立即检测甲 功和甲状腺自身抗体, 根据临床表现和FT4水平决定是否 用药：(1)停药后, 如果FT4正常, 可以继续停药, 每1 2 周进行甲功检测和临床评估, 如果FT4维持正常, 妊娠中、 晚期每2 4周检测一次, 根据检测结果, 决定用药情况; (2)停药后甲穴症状加重, 甲状腺素升高明显, 建议继续应 用抗甲状腺药物, 妊娠早期优先选择PTU, 既往应用MMI 的妊娠妇女, 若在妊娠早期需要继续治疗, 如无禁忌症, 尽快转换成PTU; (3)如果在妊娠早期之后需要继续ATD治 疗, 妊娠中、晚期是否将PTU更换为MMI, 没有明确证据, 两种药物均可能有副作用, 而且在药物转换期间可能导致 甲状腺功能波动, 不建议更改。妊娠期原则上不采取手术 治疗甲六, 如果确实需要, 手术最佳时机是妊娠中期。

妊娠合并Graves病临床甲六的ATD治疗应避免过度。 治疗后 T4低于参考范围下限的妊娠女性, 其新生儿低 $\mathrm{T} 4$ 的发生率增加, 而如果胎儿生长发育所需的 $\mathrm{T} 4$ 不足, 则不 利于胎儿发育。妊娠期监测甲六的控制指标首选血清 FT4/TT4, 控制的目标是应用最小剂量的ATD, 使血清 FT4/TT4接近或者轻度高于参考范围上限; 妊娠期应用 ATD治疗的妇女, 建议在妊娠早期检测FT4或TT4、T3和 $\mathrm{TSH}, 1$ 次/1 2周, 妊娠中、晚期 1次/2 4周, 达到目标值 后1次/4 6周。

胎儿甲状腺在妊娠 18 20周左右功能健全, 如果妊娠 早期TRAb阳性, 在妊娠18 20周左右需要检测。由于产后 新生儿清除来自母体的ATD比TRAb迅速, 新生儿可发生 甲六。因此妊娠晚期测定血清TRAb 有助于评估妊娠结局,
高滴度的 $\mathrm{TRAb}$ （高于参考范围上限3倍以上）需对胎儿行 密切随访, 与母胎医学的医生合作管理好新生儿。

\subsection{4. 妊娠合并亚临床甲光}

亚临床甲光与不良妊娠结局无明确相关性[19-21]。美 国指南指出, 抗甲状腺药物治疗对母亲或胎儿没有明显的 益处, 而且, 由于抗甲状腺药物通过胎盘可对胎儿甲状腺 功能产生不利影响, 胎儿存在一定的风险, 因此美国指南 不推荐对亚临床甲光的孕妇进行治疗。中国指南对此未进 行推荐。

\section{6. 妊娠期甲状腺结节和甲状腺癌}

妊娠妇女甲状腺结节的患病率为 $3 \% \sim 21 \%$, 且随妊娠 次数的增加而增加, 妊娠可能与己经存在的甲状腺结节大 小的增加以及新甲状腺结节形成有关[22]。美国加利福尼 亚癌症中心对当地4846505例产妇的回顾性分析发现, 甲 状腺癌在妊娠妇女中的发病率为 $14.4 / 10$ 万, 乳头状甲状腺 癌为最常见的病理类型[23]。因此对于妊娠期合并甲状腺 结节患者需要给予重视, 对此中美指南均给予了详细的推 荐及建议。

对妊娠期合并甲状腺结节患者中国指南指出要详细 询问病史、完善体格检查、测定血清TSH和颈部超声: (1) 如果TSH降低, 并持续到妊娠16周之后, 甲状腺结节细针 穿刺抽吸 (FNA) 或许可以推迟至产后进行, 如果产后TSH 仍然很低, 在不哺乳的情况下, 可行放射性核素扫描以评 估甲状腺结节功能; (2)如果TSH水平正常或升高, 应根据 结节的声像学特征决定是否做FNA, 如果良性可能性大, 可以推迟至产后进行, 如果细胞学检查为良性, 妊娠期不 需要特殊的监测; (3)妊娠早期发现的乳头状甲状腺癌, 应 每3个月复查超声, 监测肿瘤增长速度, 如果妊娠中期结 节仍然保持稳定, 或在妊娠后半期发现的结节, 手术或许 可以推迟到产后; (4)妊娠期新诊断的未分化癌或髓样癌, 对妊娠的影响尚不清楚, 然而, 治疗延迟很有可能产生不 良结局, 因此在评估所有临床因素后建议手术治疗; (5)妊 娠早期发现暂不需手术的分化型甲状腺癌（DTC），每3 个月复查超声, 监测肿瘤增长速度, 并给予LT4治疗, 治 疗目标是使血清 TSH控制在 $0.3 \sim 2.0 \mathrm{Mu} / \mathrm{L}$, 如果DTC在 24 26周前持续增大, 或发生淋巴结转移, 推荐手术治疗, DTC的手术时机应当选择在母胎风险均较小的妊娠中期 的后期。DTC患者妊娠后要定期检测血清TSH每2 4周一 次, 维持 $\mathrm{TSH}$ 在 $0.3 \sim 2.0 \mathrm{Mu} / \mathrm{L}$, 直至妊娠 20 周, $\mathrm{TSH}$ 稳定后 可每4 6周检测一次。有DTC治疗史的妇女, 如果妊娠前 不存在疾病的结构 (超声提示可疑癌结节) 或生化 (甲状 腺球蛋白 $\mathrm{Tg}$ 水平升高) 异常证据, 妊娠期不需要进行超声 和 $\mathrm{Tg}$ 监测, 若甲状腺癌治疗效果不佳, 或已知存在复发或 残留病灶, 应在妊娠期进行超声和 $\mathrm{Tg}$ 监测。

美国指南对甲状腺结节的诊疗建议相似, 但相对简单, 其中关于甲状腺碘扫描, 也不推荐在妊娠期进行。但是美 国甲状腺协会提出胎儿甲状腺在妊娠 12 周之前并没有明 显的功能活动, 如果在妊娠12周之前无意中进行了放射性 碘扫描, 胎儿甲状腺没有发现受损现象, 可考虑继续妊娠。 


\section{5. 产后甲状腺炎（PPT）}

PPT 是指妊娠前甲状腺功能正常的妇女在产后 1 年内 出现的甲状腺功能异常, 是自身免疫甲状腺炎的一个类型, $\mathrm{TPOAb}$ 或/和 $\mathrm{TgAb}$ 滴度越高, 患PPT的风险越大, PPT反映 了因妊娠而相对抑制的免疫系统在产后出现的反跳现象。 典型病例经历3期: 即甲状腺毒症期、甲减期和恢复期, 不同时期，治疗原则不同。

由于PPT是一种破坏性甲状腺炎, 甲状腺激素的合成 并未增加, 因此中国指南推荐, PPT的甲状腺毒症期不给 予ATD治疗, 可小剂量, 短疗程使用 $\beta$ 受体阻滞剂, 以减 轻症状; 甲状腺毒症期之后, 每 2 月复查一次TSH, 以及 时发现甲减并给予LT4治疗, 每4 8周复查一次TSH, 直至 甲状腺功能恢复正常; 甲减期治疗 $6 \sim 12$ 个月后, 如未哺乳, LT4开始逐渐减量, 如果正在哺乳, 暂不减少LT4的剂量。 超过 $20 \%$ 的PPT患者将发展为永久性甲减, 因此需要每年 检测 TSH, 尽早发现永久性甲减, 并给予治疗。美国指南 对此给与相似的建议。

\section{6. 妊娠期和妊娠前甲状腺疾病笁查}

由于妊娠期甲状腺激素对胎儿发育有重要影响, 因此 及早发现甲状腺疾病是孕期保健的重要部分。目前部分研 究认为, 仅针对目标病例的篮查不能达到篮查目的[24-26]。 仅在高危妊娠人群中篮查, $30 \% \sim 80 \%$ 的甲状腺疾病被漏诊。 因此如何对妊娠期甲状腺疾病进行䇻查, 显得尤为重要。

对妊娠早期妇女进行篮查的成本效益分析显示, 篮查 整个妊娠人群优于不篮查。因此中国指南推荐, 支持国内 有条件的医院和妇幼保健部门对妊娠早期妇女开展甲状 腺疾病笁查, 篮查指标选择血清TSH、FT4、TPOAb, 篮 查时机选择在妊娠 8 周以前, 最好是在妊娠前篮查[2]。

对此, 美国指南给出不一样的建议, 不推荐在妊娠期 进行甲状腺疾病普查。因为其认为识别和治疗母体亚临床 甲减或亚临床甲六并没有改善妊娠结局和后代的神经认 知功能。甲状腺功能的检测应在患有甲状腺疾病、1型糖 尿病或临床怀疑甲状腺疾病的个人或家族史的妇女中进 行。此外由于在妊娠期间甲状腺增大的发生率约 $30 \%$, 因 此美国指南认为在甲状腺轻度非对称性肿大的孕妇中进 行甲状腺功能的检测是不必要的。在有显著甲状腺肿或有 明显甲状腺结节的孕妇中, 可进行甲状腺功能检测, 因为 这些检测结果可能提示不适合妊娠。美国妇产科医师学会、 内分泌学会和美国临床内分泌学家协会建议在怀孕前对 甲状腺疾病进行篮查, 并建议在怀孕期间仅检测有明显甲 减风险的妇女[1,27]。美国甲状腺协会目前发现没有足够 的数据来推荐或反对普遍甲状腺筛查 $[28]$ 。

因此对于妊娠期和妊娠前甲状腺疾病耖查, 应从国情 出发, 最好在妊娠前进行篎查, 如已妊娠, 若条件允许可 常规进行篮查, 但如果经济条件差, 也可根据是否存在高 危因素选择性篎查。

综上所述，中美指南对妊娠期及产后甲状腺疾病的诊 疗进行了详细的分析, 虽然两指南在疾病诊疗的细节上存 在细微的差异, 少部分意见不同, 但总体建议相似, 总结 如下：对于临床甲减必须给与LT4治疗; 亚临床甲减, 根
据血清 TSH水平和 TPOAb 是否阳性给予不同的治疗方案; 妊娠期单纯低甲状腺素血症, 不推荐也不反对在妊娠早期 给予LT4治疗, 但建议查找原因, 对因治疗; 妊娠期甲状 腺自身抗体阳性, 中国指南推荐有不明原因流产史者, 给 予小剂量LT4治疗, 美国指南不推荐治疗; 妊娠期甲状腺 毒症, 必须给予相应的监测和治疗; 妊娠期甲状腺结节和 甲状腺癌, 需首先判断良恶性倾向, 良性倾向者密切随访, 辅助药物治疗, 恶性倾向者积极诊断及治疗, 甲状腺切除 手术选择妊娠中期的后期; 产后甲状腺炎, 需定期监测 $\mathrm{TSH}$, 尽早发现永久性甲减并及时治疗; 对于妊娠期和妊 娠前甲状腺疾病笁查的建议, 中国指南建议有条件者在孕 前或孕早期普遍篎查, 美国指南建议在有高危因素的患者 中进行篮查。

通过对中、美妊娠期甲状腺疾病诊疗指南的对比解读 可以达到查漏补缺、取长补短的作用, 可更好地指导临床 实践。需要注意的是, 尽管两个指南对很多问题给予了具 体的建议, 但是仍有许多问题目前尚无定论, 有待进一步 研究。同时应该建立完善的妇产科、内分泌科及甲状腺外 科等多学科协作模式, 尽量为患者提供个性化的综合治疗, 尽可能减少因甲状腺功能异常, 所导致的妊娠期相关并发 症及胎儿的近远期影响, 提高出生人口素质。

\section{参考文献}

[1] De Groot L, Abalovich M, Alexander EK, et al. Management of thyroid dysfunction during pregnancy and postpartum: an Endocrine Society clinical practice guideline. J Clin Endocrinol Metab, 2012, 97(8): 2543-2565.

[2]《妊娠和产后甲状腺疾病诊治指南》编撰委员会, 中华医 学会内分泌学分会, 中华医学会围产医学分会. 妊娠和产 后甲状腺疾病诊治指南(第2版). 中华内分泌代谢杂志, 2019, 35(8): 636-665.

[3] Thyroid Disease in Pregnancy: ACOG Practice Bulletin, Number 223. Obstet Gynecol, 2020, 135(6): e261-e274.

[4] Vannucchi G, Covelli D, Vigo B, et al. Thyroid volume and serum calcitonin changes during pregnancy. Journal of Endocrinological Investigation, 2017, 40(7): 727-732.

[5] Li C, Shan Z, Mao J, et al. Assessment of thyroid function during first-trimester pregnancy: what is the rational upper limit of serum TSH during the first trimester in Chinese pregnant women? J Clin Endocrinol Metab, 2014, 99(1): 73-79.

[6] Cleary-Goldman J, Malone FD, Lambert-Messerlian G, et al. Maternal thyroid hypofunction and pregnancy outcome. Obstet Gynecol, 2008, 112(1): 85-92.

[7] Hales C, Taylor PN, Channon S, et al. Controlled Antenatal Thyroid Screening II: Effect of Treating Maternal Suboptimal Thyroid Function on Child Cognition. J Clin Endocrinol Metab, 2018, 103(4): 1583-1591.

[8] Zhang Y, Wang H, Pan X, et al. Patients with subclinical hypothyroidism before 20 weeks of pregnancy have a higher risk of miscarriage: A systematic review and meta-analysis. PLoS One, 2017, 12(4): e0175708. 
[9] Shi X, Han C, Li C, et al. Optimal and safe upper limits of iodine intake for early pregnancy in iodine-sufficient regions: a cross-sectional study of 7190 pregnant women in China. J Clin Endocrinol Metab, 2015, 100(4): 1630-1638.

[10] Teng X, Shan Z, Li C, et al. Iron Deficiency May Predict Greater Risk for Hypothyroxinemia: A Retrospective Cohort Study of Pregnant Women in China. Thyroid, 2018, 28(8): 968-975.

[11] Thangaratinam S, Tan A, Knox E, et al. Association between thyroid autoantibodies and miscarriage and preterm birth: meta-analysis of evidence. Bmj, 2011, 342: d2616.

[12] Andersen SL, Olsen J, Laurberg P. Foetal programming by maternal thyroid disease. Clinical Endocrinology, 2015, 83(6):751-758.

[13] Korevaar TIM, Muetzel R, Medici M, et al. Association of maternal thyroid function during early pregnancy with offspring IQ and brain morphology in childhood: a population-based prospective cohort study. Lancet Diabetes \& Endocrinology, 2016, 4(1):35-43.

[14] Donnelly MA, Wood C, Casey B, et al. Early severe fetal Graves disease in a mother after thyroid ablation and thyroidectomy. Obstet Gynecol, 2015, 125(5): 1059-1062.

[15] Andersen SL, Olsen J, Wu CS, et al. Birth defects after early pregnancy use of antithyroid drugs: a Danish nationwide study. J Clin Endocrinol Metab, 2013, 98(11): 4373-4381.

[16] Seo GH, Kim TH, Chung JH. Antithyroid Drugs and Congenital Malformations: A Nationwide Korean Cohort Study. Ann Intern Med, 2018, 168(6): 405-413.

[17] Maurizio C, Di GE, Matteo C, et al. Treatment of Hyperthyroidism in Pregnancy and Birth Defects. Journal of Clinical Endocrinology \& Metabolism, 2010, (11): 11.

[18] Ai Y, JaedukYoshimura N, Takuhiro Y, et al. Treatment of Graves' Disease with Antithyroid Drugs in the First Trimester of Pregnancy and the Prevalence of Congenital Malformation. Journal of Clinical Endocrinology \& Metabolism, 2012, (7): 7.
[19] Tudela CM, Casey BM, Mcintire DD, et al. Relationship of Subclinical Thyroid Disease to the Incidence of Gestational Diabetes. Obstetrics \& Gynecology, 2012, 119(5): 983-988.

[20] Wilson KL. Subclinical thyroid disease and the incidence of hypertension in pregnancy. Obstetrics \& Gynecology, 2012, 119(1): 315-320.

[21] Casey BM, Dashe JS, Wells CE, et al. Subclinical Hyperthyroidism and Pregnancy Outcomes. Obstetrics \& Gynecology, 2006, 107(2, Part 1): 337-341.

[22] Kung AWC, Chau MT, Lao TT, et al. The effect of pregnancy on thyroid nodule formation. Journal of Clinical Endocrinology \& Metabolism, 2002, 87(3): 1010-1014.

[23] Smith LH, Danielsen B, Allen ME, et al. Cancer associated with obstetric delivery: results of linkage with the California cancer registry. American Journal of Obstetrics \& Gynecology, 2003, 189(4): 1128-1135.

[24] Vaidya B, Anthony S, Bilous M, et al. Detection of thyroid dysfunction in early pregnancy: Universal screening or targeted high-risk case finding? J Clin Endocrinol Metab, 2007, 92(1): 203-207.

[25] Wang W, Teng W, Shan Z, et al. The prevalence of thyroid disorders during early pregnancy in China: the benefits of universal screening in the first trimester of pregnancy. European Journal of Endocrinology, 2011, 164(2): 263-268.

[26] Hong, Yang, Minglong, et al. Screening Strategies for Thyroid Disorders in the First and Second Trimester of Pregnancy in China. Plos One, 2014,9(6):e99611.

[27] Garber JR, Cobin RH, Gharib H, et al. Clinical practice guidelines for hypothyroidism in adults: cosponsored by the American Association of Clinical Endocrinologists and the American Thyroid Association. Thyroid, 2012, 22(12): 1200-1235.

[28] Alexander EK, Pearce EN, Brent GA, et al. 2017 Guidelines of the American Thyroid Association for the Diagnosis and Management of Thyroid Disease During Pregnancy and the Postpartum. Thyroid, 2017, 27(3): 315-389. 